\section{Autoantikörper gegen F-Actin}

\author{
W. Stöcker \\ Euroimmun Medizinische Labordiagnostika AG, Lübeck, \\ Deutschland
}

Synonym(e) Autoantikörper gegen filamentöses Actin (F-Actin); Anti-F-Actin-Antikörper

Englischer Begriff autoantibodies against filamentous actin (F-actin)

Definition Autoantikörper gegen filamentöses Actin (F-Actin) des zytoskelettalen Mikrofilaments und der Muskelfasern sind eine Untergruppe der $>$ Autoantikörper gegen glatte Muskulatur (,,anti-smooth muscle antibody“, ASMA). Im Gegensatz zu anderen ASMA sind Autoantikörper gegen F-Actin sehr spezifische Marker für eine Autoimmunhepatitis (AIH) vom Typ I.

Funktion - Pathophysiologie Hohe Konzentrationen der Autoantikörper gegen glatte Muskeln weisen auf eine Autoimmunhepatitis (AIH) hin. Ein Teil der Antikörper richtet sich gegen Konformationsepitope des F-Actins. Als molekulare Ziele der Autoantikörper gegen F-Actin wurden neben dem Actin auch Actin-bindende Proteine wie Filamin, Actinin oder Tropomyosin beschrieben.

Analytik Autoantikörper gegen F-Actin lassen sich durch indirekte Immunfluoreszenz ( Immunfluoreszenz, indirekte) mit Zellen der vaskulären glatten Muskulatur (VSM47) als Substrat bestimmen und sind an dem typischen mikrofilamentösen Fluoreszenzmuster zu erkennen. Sie fär- ben auch typischerweise die Gefäßwände (glatte Muskulatur) sowie die Glomeruli und Tubuli der Niere an.

\section{Untersuchungsmaterial Serum.}

Probenstabilität Autoantikörper sind bei $+4{ }^{\circ} \mathrm{C}$ bis $\mathrm{zu}$ 2 Wochen lang beständig, bei $-20^{\circ} \mathrm{C}$ über Monate und Jahre hinweg.

Diagnostische Wertigkeit Die Bestimmung der Autoantikörper gegen F-Actin ist von besonderer Bedeutung für die Diagnose der Autoimmunhepatitis (AIH, Prävalenz etwa 50 \%), den Ausschluss einer kombinierten Lebererkrankung (Overlap-Syndrom) und die Abgrenzung der AIH gegenüber einer Alkohol- oder Medikamenten-induzierten Zirrhose und anderen chronischen Entzündungen der Leber, wie Virusinduzierte Hepatitis, primär-biliäre Cholangitis ( $\mathrm{PBC}$, früher: Primär biliäre Zirrhose) und primär-sklerosierende Cholangiitis (PSC; s. a. > Autoantikörper gegen glatte Muskulatur).

\section{Literatur}

Czaja AJ (2007) Autoimmune liver diseases. Curr Opin Gastroenterol 23:255-262

Villalta D, Bizzaro N, Da Re M, Tozzoli R, Komorowski L, Tonutti E (2008) Diagnostic accuracy of four different immunological methods for the detection of anti-F-actin autoantibodies in type 1 autoimmune hepatitis and other liver-related disorders. Autoimmunity $41: 105-110$

Villalta D, Girolami E, Alessio MG, Sorrentina MC, Tampoia M, Brusca I, Daves M, Porcelli B, Barberio G, Conte M, Pantarotto L, Bizzaro N, Study Group on Autoimmune Diseases of the Italian Society of Laboratory Medicine, Italy (2016) Autoantibody profiling in a cohort of pediatric and adult patients with autoimmune hepatitis. J Clin Lab Anal 30(1):41-46 